\title{
Simultaneous Nulling for Monopulse Array with Partially Adaptive Weights
}

\author{
Ta-Sung Lee, Member, IEEE
}

\begin{abstract}
A new simple method is described for synthesizing low-sidelobe sum and difference patterns with partially adaptive weights. By partially adaptive, we mean that only part of the weights are adapted for simultaneous nulling. These adaptive weights are shared by the sum and difference channels, leading to a significant reduction in the number of variable attenuators/phase shifters used, as compared to the fully adaptive implementation. An objective function is derived that yields different configurations of the shared adaptive weights. Numerical examples are presented to ascertain the efficacy of the new method for both point and extended interference.
\end{abstract}

\section{INTRODUCTION}

$\mathbf{S}$ IMULTANEOUS nulling is essential for a monopulse radar operated in an environment contaminated with strong interference. With the advent of modern phased array technology, simultaneous nulling can be accomplished via the adaptation of two independent sets of complex weights, one for the sum channel and the other for the difference channel [1], [2]. In spite of its flexibility, adaptive nulling with full amplitude and phase control for both channels is rather expensive, considering the cost of phase shifters and variable attenuators. As a remedy, the ideas of phase-only control [3], [4] and amplitude-only control [5] were proposed; they allow one to synthesize the desired sum and difference patterns using fixed amplitude weights or real weights. The drawback of phase-only implementation is that the numerical complexity involved in solving for the phase adjustments is high, except for the case of small perturbation in which the problem can be linearized. Amplitude-only implementation is simple, but it usually results in undesired pattern shapes. In another approach to simplifying the hardware, the sum and difference channels share the same set of complex weights, with the sign reversed for half the weights for the difference channel [5]. Its drawback is that the difference pattern exhibits undesired high sidelobes due to the discontinuity of the taper at the center of the aperture. A different approach was suggested [1] based on the use of shared weight perturbations, i.e., the same set of amplitude and phase perturbations are imposed on the preselected quiescent sum and difference weights. Although the use of shared weight perturbations achieves good performance, it increases the hardware complexity as compared to the shared weight implementation. Subarray

Manuscript received February 24, 1994; revised January 18, 1994. This work was supported by the National Science Council of R.O.C. under Grant NSC 82-0404-E-009-333.

The author is with the Department of Communication Engineering, National Chiao Tung University, Hsinchu, Taiwan, Republic of China.

IEEE Log Number 9402851 beamforming was also proposed [6] as an economical means of adaptive nulling. In this approach, adaptive weights are placed at the subarray outputs, resulting in fewer variable attenuators. Unfortunately, grating lobe problems limit this form of implementation.

We here present a new simple method for simultaneous nulling with low-sidelobe sum and difference patterns using a uniform, linear array (ULA). The method is prompted by the fact that the Chebyshev and Bayliss tapers are similar in shape near both ends of the aperture. It is thus reasonable to use a common set of complex weights for both channels at the tail portions. To reduce hardware complexity, the amplitude weights at the center portions of the aperture are fixed for both channels, and are copied from the desired sum (Chebyshev) and difference (Bayliss) tapers. Fixing the center amplitude weights in this fashion offers the advantage of preserving the shapes of the desired patterns under adaptive nulling operations. Owing to the reduction of free complex weights, the maximum number of individual point interferers that can be nulled exactly is decreased. To remedy this, phase-only adaptation is incorporated on the unshared center weights for both channels. Introducing phase-only adaptation provides an extra degree of freedom for improving the sidelobe behaviors of the synthesized patterns. To avoid high numerical complexity, we manage to keep the phase perturbations small so that first-order approximation can be used. An objective function is set up for obtaining the optimum weight vectors that produce the sum and difference patterns best approximating the desired ones in the sense of minimum $L_{2}$ distance. By choosing proper weighting factors in the objective function, various configurations of the shared adaptive weights are obtained. Numerical examples confirm that the proposed method can indeed perform simultaneous nulling, while keeping the sidelobe level low enough as is desired.

\section{NOTATIONS AND MODEL FORMULATION}

Some of the notations used in the paper are defined as follows:

$z^{*}$ : complex conjugate of $z$.

$M^{T}\left(v^{T}\right)$ : transpose of matrix $M$ (vector $v$ ).

$\boldsymbol{M}^{H}\left(\boldsymbol{v}^{H}\right)$ : conjugate transpose of matrix $\boldsymbol{M}$ (vector $\boldsymbol{v}$ ).

$I_{n}: n \times n$ identity matrix.

$\tilde{I}_{n}: n \times n$ reverse permutation matrix with ones on the antidiagonal and zeros elsewhere. It reverses the columns of the matrix premultiplied by it.

$O_{m \times n}: m \times n$ zero matrix. 
$\mathbf{o}_{n}: n \times 1$ zero vector.

$\operatorname{Diag}\{\boldsymbol{v}\}$ : diagonal matrix with its diagonal components given by vector $\boldsymbol{v}$.

Consider a linear array consisting of $M$ identical elements uniformly spaced by a half wavelength. The far field pattern of the array associated with a set of complex weights $w_{i}$, $i=1, \cdots, M$, can be expressed as

$$
w(u)=\boldsymbol{w}^{H} \boldsymbol{a}(u)
$$

where

$$
\boldsymbol{w}=\left[w_{1}, w_{2}, \cdots, w_{M}\right]^{T}
$$

is the $M \times 1$ weight vector, and

$$
\begin{aligned}
& \boldsymbol{a}(u)= \\
& \quad\left[e^{-j \pi\left(\frac{M-1}{2}\right) u}, e^{-j \pi\left(\frac{M-3}{2}\right) u}, \cdots, e^{j \pi\left(\frac{M-3}{2}\right) u}, e^{j \pi\left(\frac{M-1}{2}\right) u}\right]^{T}
\end{aligned}
$$

is the $M \times 1$ array response vector accounting for the phase variation across the array. The variable $u=\sin (\theta)$ is the sinespace angle, with $\theta$ representing the physical angle with respect to the broadside of the array. For the case of half-wavelength spacing, $u$ and $\theta$ are uniquely related over $-1 \leq u \leq 1$, or $-90^{\circ} \leq \theta \leq 90^{\circ}$. Note that we have set the reference point of the array to be at its geometric center such that $\boldsymbol{a}(u)$ is conjugate symmetric, i.e., $\tilde{\boldsymbol{I}}_{M} \boldsymbol{a}(u)=\boldsymbol{a}^{*}(u)$. As a consequence, if the weight vector is also conjugate symmetric, then the pattern will be purely real, which is a desired property in monopulse operation. In the following development, we will assume that all the weight vectors referred to are conjugate symmetric.

A criterion for choosing the sum and difference weights in monopulse arrays is the capability of suppressing interference from outside the mainlobe region. To ensure the protection from interference in all directions, patterns with uniformly low sidelobes are desired. Also, to enhance the accuracy of target bearing estimation, a narrow mainlobe for the sum pattern and a large boresight slope for the difference pattern are desired. The Chebyshev taper [7] and the Bayliss taper [8] are the appropriate candidates for the sum and difference channels, respectively, considering the aforementioned mainlobe and sidelobe behaviors. In this paper, an efficient and cheap implementation of the adaptive sum and difference beamformers is proposed that retains the desired properties of the Chebyshev and Bayliss beamformers under simultaneous nulling operations.

\section{PARTIAL Shared WeIGHTS IMPleMENTATION}

A simple way to implement the sum and difference beamformers is to determine the weights for the sum channel first, and then reverse the sign of half the weights for the difference channel. The simplification in system complexity is that only a single set of complex weights is needed. However, working with full shared weights usually results in poor sidelobe behaviors due to the discontinuity of the difference taper. As a remedy, we propose the use of partial shared weights.

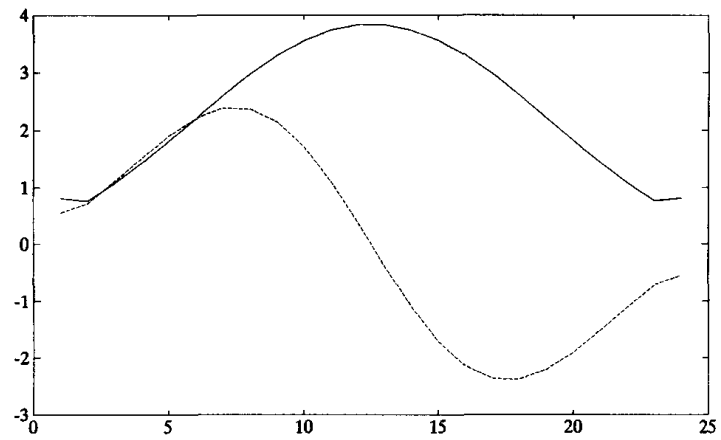

Fig. 1. Normalized 24-point Chebyshev and Bayliss tapers with $-35 \mathrm{~dB}$ sidelobes. Solid line: Chebyshev; dashed line: Bayliss.

For the sake of convenience, we will assume that $M$ is even and the boresight angle is zero. For a nonzero boresight angle, the results are easily modified with an appropriate progressive phase shifting. To demonstrate how the shared weights should be chosen, we consider the shapes of the Chebyshev and Bayliss tapers, as shown in Fig. 1. The Chebyshev taper resembles a half cycle cosine wave, whereas the Bayliss taper resembles a full cycle sine wave. The two tapers are quite different at the center, but are similar in shape at the tails. This prompts us to implement the sum and difference beamformers sharing the same $J<\frac{M}{2}$ weights from both ends of the aperture. Incorporation of this constraint and imposing conjugate symmetry leads to the following segmental structures for the sum and difference weight vectors:

$$
\begin{gathered}
\boldsymbol{s}=\left[\begin{array}{c}
\boldsymbol{r} \\
\boldsymbol{c}_{\boldsymbol{s}} \\
\tilde{\boldsymbol{I}}_{N} \boldsymbol{c}_{\boldsymbol{s}}^{*} \\
\tilde{\boldsymbol{I}}_{J} \boldsymbol{r}^{*}
\end{array}\right], \\
\boldsymbol{d}=\left[\begin{array}{c}
j \boldsymbol{r} \\
j \boldsymbol{c}_{d} \\
-j \tilde{\boldsymbol{I}}_{N} \boldsymbol{c}_{d}^{*} \\
-j \tilde{\boldsymbol{I}}_{J} \boldsymbol{r}^{*}
\end{array}\right]
\end{gathered}
$$

where $\boldsymbol{r}$ is the $J \times 1$ complex vector representing half the shared tail weights. $c_{s}$ and $c_{d}$ are the $N \times 1\left(N=\frac{M-2 J}{2}\right)$ complex vectors representing half the unshared center weights associated with the sum and difference channels, respectively. Observing Fig. 1 again indicates that the appropriate configuration should be $J \approx \frac{M}{4}$. Similarly, we can express the Chebyshev and Bayliss weight vectors in accordance with (4):

$$
\begin{gathered}
\boldsymbol{s}_{o}=\left[\begin{array}{c}
\boldsymbol{r}_{s o} \\
\boldsymbol{c}_{s o} \\
\tilde{\boldsymbol{I}}_{N} \boldsymbol{c}_{s o} \\
\tilde{\boldsymbol{I}}_{J} \boldsymbol{\tau}_{s o}
\end{array}\right], \\
\boldsymbol{d}_{o}=\left[\begin{array}{c}
j \boldsymbol{r}_{d o} \\
j \boldsymbol{c}_{d o} \\
-j \tilde{\boldsymbol{I}}_{N} \boldsymbol{c}_{d o} \\
-j \tilde{\boldsymbol{I}}_{J} \boldsymbol{r}_{d o}
\end{array}\right]
\end{gathered}
$$

where we note that the subvectors $\boldsymbol{r}_{s o}, \boldsymbol{c}_{s o}, \boldsymbol{r}_{d o}$ and $\boldsymbol{c}_{d o}$ are all real. 
To simplify the hardware complexity, the amplitudes of the center weights $c_{s}$ and $c_{d}$ are fixed and copied directly from $c_{s o}$ and $c_{d o}$, respectively. This should help to retain the desired low sidelobe behavior for $\boldsymbol{s}$ and $\boldsymbol{d}$. However, employing fixed center amplitude weights reduces the degree of freedom in nulling point interferers. To remedy this, phase-only adaptation is incorporated on the center weights. Introducing phase-only adaptation not only provides an extra degree of freedom for nulling but also improves the sidelobe behaviors of the synthesized patterns. The drawback of phase-only implementation is the high numerical complexity incurred with the nonlinearity of the problem. In some cases, it is assumed that the phase perturbations are small such that the exponentials can be linearized [3], [4]. The small perturbation assumption holds typically when the number of adaptive nulls is small relative to the number of independent weights, and when the perturbed patterns are close to the original ones. A scheme will be presented shortly that ensures that the phase perturbations are small enough.

In the proposed method, the same set of phase perturbations are imposed on the center weights. Since the tail weights are already shared, this would require only a single set of variable phase shifters for both channels. Let $\phi_{k}, k=1, \cdots, 2 N$, be these phase perturbations. Under the small perturbation assumption, we have $e^{j \phi_{k}} \approx 1+j \phi_{k}, k=1, \cdots, 2 N$, such that

$$
\begin{aligned}
& c_{s} \approx c_{s o}+j C_{s o} \phi, \\
& c_{d} \approx c_{d o}+j C_{d o} \phi
\end{aligned}
$$

where $\boldsymbol{C}_{\text {so }}=\operatorname{Diag}\left\{\boldsymbol{c}_{s o}\right\}, \boldsymbol{C}_{d o}=\operatorname{Diag}\left\{\boldsymbol{c}_{d o}\right\}$ and

$$
\boldsymbol{\phi}=\left[\phi_{1}, \phi_{2}, \cdots, \phi_{N}\right]^{T} \text {. }
$$

Because of the conjugate symmetry of the weight vectors, we have $\phi_{k}=-\phi_{2 N-k+1}, k=1, \cdots, N$, such that there are only $N$ independent phase perturbations, as given by (7). Combining (4) and (6) yields the expressions for $s$ and $\boldsymbol{d}$ decomposed into the variable and fixed parts:

$$
\begin{gathered}
\boldsymbol{s}=\left[\begin{array}{c}
\boldsymbol{r} \\
j \boldsymbol{C}_{s o} \phi \\
-j \tilde{\boldsymbol{I}}_{N} \boldsymbol{C}_{s o} \boldsymbol{\phi} \\
\tilde{\boldsymbol{I}}_{J} \boldsymbol{r}^{*}
\end{array}\right]+\left[\begin{array}{c}
\mathbf{o}_{J} \\
\boldsymbol{c}_{s o} \\
\tilde{\boldsymbol{I}}_{N} \boldsymbol{c}_{s o} \\
\mathbf{o}_{J}
\end{array}\right], \\
\boldsymbol{d}=\left[\begin{array}{c}
j \boldsymbol{r} \\
-\boldsymbol{C}_{d o} \boldsymbol{\phi} \\
-\tilde{\boldsymbol{I}}_{N} \boldsymbol{C}_{d o} \boldsymbol{\phi} \\
-j \tilde{\boldsymbol{I}}_{J} \boldsymbol{r}^{*}
\end{array}\right]+\left[\begin{array}{c}
\mathbf{o}_{J} \\
j \boldsymbol{c}_{d o} \\
-j \tilde{\boldsymbol{I}}_{N} c_{d o} \\
\mathbf{o}_{J}
\end{array}\right] .
\end{gathered}
$$

\section{A. Adaptive Interference Cancellation}

In the presence of strong out-of-band active interferers, the sidelobes of the sum and difference patterns may not be low enough to provide effective suppression of these undesired sources. It is then necessary to perform adaptive cancellation by putting a deep null in the direction of each of the interferers for the sum and difference patterns. Assume that the interfering directions are first estimated during the passive period of the radar via some kind of off-line direction finding algorithm. The sum and difference patterns are then synthesized accordingly to put "hard nulls" in these directions.

Let $u_{i}, i=1, \cdots, K$, be the $K$ estimated interfering directions. The execution of interference cancellation requires

$$
s\left(u_{i}\right)=d\left(u_{i}\right)=0 \quad i=1, \cdots, K
$$

where $s(u)=\boldsymbol{s}^{H} \boldsymbol{a}(u)$ and $d(u)=\boldsymbol{d}^{H} \boldsymbol{a}(u)$ are the sum and difference patterns, respectively. Rewriting (9) in matrix form yields

$$
\boldsymbol{A}^{H} \boldsymbol{s}=\boldsymbol{A}^{H} \boldsymbol{d}=\mathbf{o}_{K}
$$

where

$\boldsymbol{A}=\left[\begin{array}{llll}\boldsymbol{a}\left(u_{1}\right) & \boldsymbol{a}\left(u_{2}\right) & \cdots & \boldsymbol{a}\left(u_{K}\right)\end{array}\right]=\left[\begin{array}{c}\boldsymbol{A}_{r} \\ \boldsymbol{A}_{c} \\ \tilde{\boldsymbol{I}}_{N} \boldsymbol{A}_{c}^{*} \\ \tilde{\boldsymbol{I}}_{J} \boldsymbol{A}_{r}^{*}\end{array}\right] \begin{gathered}J \times K \\ N \times K \\ N \times K \\ J \times K\end{gathered}$

is the $M \times K$ interference response matrix partitioned in accordance with (4). Substituting (8) and (11) into (10) and using $\tilde{I}_{J}^{2}=I_{J}$ and $\tilde{I}_{N}^{2}=I_{N}$ leads to the following systems of equations:

$$
\begin{gathered}
\boldsymbol{A}_{r}^{H} \boldsymbol{r}+\boldsymbol{A}_{r}^{T} \boldsymbol{r}^{*}+j\left(\boldsymbol{A}_{c}^{H}-\boldsymbol{A}_{c}^{T}\right) \boldsymbol{C}_{s o} \boldsymbol{\phi}=-\left(\boldsymbol{A}_{c}^{H}+\boldsymbol{A}_{c}^{T}\right) \boldsymbol{c}_{s o} \\
j\left(\boldsymbol{A}_{r}^{H} \boldsymbol{r}-\boldsymbol{A}_{r}^{T} \boldsymbol{r}^{*}\right)-\left(\boldsymbol{A}_{c}^{H}+\boldsymbol{A}_{c}^{T}\right) \boldsymbol{C}_{d o} \boldsymbol{\phi}=-j\left(\boldsymbol{A}_{c}^{H}-\boldsymbol{A}_{c}^{T}\right) \boldsymbol{c}_{d o} .
\end{gathered}
$$

It is a straightforward matter to verify that the two equations in (12) can be combined into a real system of equations of the form:

$$
E x=g
$$

where

$$
\boldsymbol{E}=\left[\begin{array}{ccc}
\operatorname{Re}\left\{\boldsymbol{A}_{r}^{H}\right\} & -\operatorname{Im}\left\{\boldsymbol{A}_{r}^{H}\right\} & -\operatorname{Im}\left\{\boldsymbol{A}_{c}^{H}\right\} \boldsymbol{C}_{s o} \\
\operatorname{Im}\left\{\boldsymbol{A}_{r}^{H}\right\} & \operatorname{Re}\left\{\boldsymbol{A}_{r}^{H}\right\} & \operatorname{Re}\left\{\boldsymbol{A}_{c}^{H}\right\} \boldsymbol{C}_{d o}
\end{array}\right]
$$

is $2 K \times(N+2 J)$,

$$
\boldsymbol{x}=\left[\begin{array}{c}
\operatorname{Re}\{\boldsymbol{r}\} \\
\operatorname{Im}\{\boldsymbol{r}\} \\
\boldsymbol{\phi}
\end{array}\right]
$$

and

$$
\boldsymbol{g}=-\left[\begin{array}{l}
\operatorname{Re}\left\{\boldsymbol{A}_{c}^{H} \boldsymbol{c}_{\boldsymbol{s o}}\right\} \\
\operatorname{Im}\left\{\boldsymbol{A}_{c}^{H} c_{d o}\right\}
\end{array}\right]
$$

with $\operatorname{Re}\{\cdot\}$ and $\operatorname{Im}\{\cdot\}$ denoting the real and imaginary parts, respectively. In general, (13) does not have an exact solution for $x$ if $K>\frac{N+2 J}{2}=\frac{M+2 J}{4}$, meaning that the maximum number of interferers which can be nulled is reduced to $\frac{M+2 J}{4}$. 


\section{B. Underdetermined Case}

For $K<\frac{N+2 J}{2},(13)$ is an underdetermined system of equations whose solution is given by

$$
\boldsymbol{x}=\boldsymbol{E}^{+} \boldsymbol{g}+\overline{\boldsymbol{E}} \boldsymbol{y}
$$

where

$$
\boldsymbol{E}^{+}=\boldsymbol{E}^{H}\left(\boldsymbol{E} \boldsymbol{E}^{H}\right)^{-1}
$$

is the right pseudo inverse of $\boldsymbol{E}, \overline{\boldsymbol{E}}$ is an $(N+2 J) \times(N+2 J-$ $2 K$ ) matrix having as columns the vectors spanning the null space of $\boldsymbol{E}$, and $\boldsymbol{y}$ is an arbitrary real vector of a compatible size.

Since our goal is to retain the shapes of the Chebyshev and the Bayliss patterns, a reasonable and convenient way to determine the free vector $y$ would be to form the LS-fit problem:

$$
\begin{aligned}
\min _{\boldsymbol{y}} \int_{-1}^{1}\left\{\left[s(u)-s_{o}(u)\right]^{2}+\right. & {\left.\left[d(u)-d_{o}(u)\right]^{2}\right\} d u } \\
= & \left\|\boldsymbol{s}-\boldsymbol{s}_{o}\right\|^{2}+\left\|\boldsymbol{d}-\boldsymbol{d}_{o}\right\|^{2}
\end{aligned}
$$

where $\|\cdot\|$ denotes the vector 2-norm. $s_{o}(u)$ and $d_{o}(u)$ are the Chebyshev and Bayliss patterns obtained with $\boldsymbol{s}_{o}$ and $\boldsymbol{d}_{o}$, respectively. Note that in (19) we have invoked the Parseval's relationship for ULA. Substitution of (5), (8), and (17) into (19), with the separation of shared/unshared weights and real/imaginary parts, leads to

$\min _{\boldsymbol{y}}\left\|\left[\begin{array}{c}\boldsymbol{r}-\boldsymbol{r}_{s o} \\ j \boldsymbol{C}_{s o} \boldsymbol{\phi} \\ -j \tilde{\boldsymbol{I}}_{N} \boldsymbol{C}_{s o} \boldsymbol{\phi} \\ \tilde{\boldsymbol{I}}_{J}\left(\boldsymbol{r}^{*}-\boldsymbol{r}_{s o}\right)\end{array}\right]\right\|^{2}+\left\|\left[\begin{array}{c}j\left(\boldsymbol{r}-\boldsymbol{r}_{d o}\right) \\ \boldsymbol{C}_{d o} \boldsymbol{\phi} \\ -\tilde{\boldsymbol{I}}_{N} \boldsymbol{C}_{d o} \boldsymbol{\phi} \\ -j \tilde{\boldsymbol{I}}_{J}\left(\boldsymbol{r}^{*}-\boldsymbol{r}_{d o}\right)\end{array}\right]\right\|^{2}$

$=2\left\|\left[\begin{array}{c}r-r_{s o} \\ C_{s o} \phi\end{array}\right]\right\|^{2}+2\left\|\left[\begin{array}{c}r-r_{d o} \\ C_{d o} \phi\end{array}\right]\right\|^{2}$

$=2\left\|\left[\begin{array}{c}\operatorname{Re}\{\boldsymbol{r}\}-\boldsymbol{r}_{s o} \\ \operatorname{Im}\{\boldsymbol{r}\} \\ \boldsymbol{C}_{s o} \boldsymbol{\phi}\end{array}\right]\right\|^{2}+2\left\|\left[\begin{array}{c}\operatorname{Re}\{\boldsymbol{r}\}-\boldsymbol{r}_{d o} \\ I m\{\boldsymbol{r}\} \\ \boldsymbol{C}_{d o} \boldsymbol{\phi}\end{array}\right]\right\|^{2}$

$=2\left\|\boldsymbol{T}_{s} \boldsymbol{x}-\tilde{\boldsymbol{r}}_{s o}\right\|^{2}+2\left\|\boldsymbol{T}_{d} \boldsymbol{x}-\tilde{\boldsymbol{r}}_{d o}\right\|^{2}$

$=2\left\|\boldsymbol{T}_{s} \overline{\boldsymbol{E}} \boldsymbol{y}+\boldsymbol{T}_{\boldsymbol{s}} \boldsymbol{E}^{+} \boldsymbol{g}-\tilde{\boldsymbol{r}}_{s o}\right\|^{2}+2\left\|\boldsymbol{T}_{d} \overline{\boldsymbol{E}} \boldsymbol{y}+\boldsymbol{T}_{d} \boldsymbol{E}^{+} \boldsymbol{g}-\tilde{\boldsymbol{r}}_{d o}\right\|^{2}$

$=2\|\boldsymbol{F} \boldsymbol{y}-\boldsymbol{h}\|^{2}$

where

$$
\begin{aligned}
\boldsymbol{T}_{s} & =\left[\begin{array}{cc}
\boldsymbol{I}_{2 J} & \boldsymbol{O}_{2 J \times N} \\
\boldsymbol{O}_{N \times 2 J} & \boldsymbol{C}_{s o}
\end{array}\right] \\
\boldsymbol{T}_{d} & =\left[\begin{array}{cc}
\boldsymbol{I}_{2 J} & \boldsymbol{O}_{2 J \times N} \\
\boldsymbol{O}_{N \times 2 J} & \boldsymbol{C}_{d o}
\end{array}\right] \\
\tilde{\boldsymbol{r}}_{s o} & =\left[\begin{array}{l}
\boldsymbol{r}_{s o} \\
\mathbf{o}_{\frac{M}{2}}
\end{array}\right] \\
\tilde{\boldsymbol{r}}_{d o} & =\left[\begin{array}{l}
\boldsymbol{r}_{d o} \\
\mathbf{o}_{\frac{M}{2}}^{2}
\end{array}\right] \\
\boldsymbol{F} & =\left[\begin{array}{l}
\boldsymbol{T}_{s} \overline{\boldsymbol{E}} \\
\boldsymbol{T}_{d} \overline{\boldsymbol{E}}
\end{array}\right]
\end{aligned}
$$

and

$$
\boldsymbol{h}=\left[\begin{array}{l}
\tilde{\boldsymbol{r}}_{s o}-\boldsymbol{T}_{s} \boldsymbol{E}^{+} \boldsymbol{g} \\
\tilde{\boldsymbol{r}}_{d o}-\boldsymbol{T}_{d} \boldsymbol{E}^{+} \boldsymbol{g}
\end{array}\right]
$$

Note that we have used the following identities,

$$
\begin{gathered}
\left\|\left[\begin{array}{c} 
\pm \boldsymbol{v} \\
\pm j \tilde{\boldsymbol{I}} \boldsymbol{v}^{*}
\end{array}\right]\right\|^{2}=2\|\boldsymbol{v}\|^{2} \\
\|\boldsymbol{v}\|^{2}=\left\|\left[\begin{array}{c}
\operatorname{Re}\{\boldsymbol{v}\} \\
\operatorname{Im}\{\boldsymbol{v}\}
\end{array}\right]\right\|^{2}, \\
\left\|\boldsymbol{v}_{1}\right\|^{2}+\left\|\boldsymbol{v}_{2}\right\|^{2}=\left\|\left[\begin{array}{l}
\boldsymbol{v}_{1} \\
\boldsymbol{v}_{2}
\end{array}\right]\right\|^{2}
\end{gathered}
$$

and the fact that $\boldsymbol{r}_{s_{0}}$ and $\boldsymbol{r}_{d o}$ are both real in obtaining the equalities in (20). Since $\boldsymbol{F}$ is a $(2 N+4 J) \times(N+2 J-2 K)$ full rank matrix, the solution to $(20)$ is given by

$$
\boldsymbol{y}=\left(\boldsymbol{F}^{H} \boldsymbol{F}\right)^{-1} \boldsymbol{F}^{H} \boldsymbol{h} \text {. }
$$

Substituting (26) back into (17) then yields the final form of $x$.

\section{Modified LS-Fit Objective Function}

The validity of linearization according to (6) lies in that the $M-2 J$ phase perturbations on the center weights are much smaller than one. When this is not the case, the synthesized patterns may not exhibit nulls exactly at, or at least close to, the specified interfering directions. A remedy would be to incorporate a weighting factor in the LS-fit objective function (20) to emphasize the errors in the center weights for both channels. This is accomplished by modifying (20) into

$$
\min _{\boldsymbol{y}}\left\|\left[\begin{array}{c}
\operatorname{Re}\{\boldsymbol{r}\}-\boldsymbol{r}_{s o} \\
\operatorname{Im}\{\boldsymbol{r}\} \\
\boldsymbol{P}_{c} \boldsymbol{C}_{s o} \boldsymbol{\phi}
\end{array}\right]\right\|^{2}+\left\|\left[\begin{array}{c}
\operatorname{Re}\{\boldsymbol{r}\}-\boldsymbol{r}_{d o} \\
I m\{\boldsymbol{r}\} \\
\boldsymbol{P}_{c} \boldsymbol{C}_{d o} \boldsymbol{\phi}
\end{array}\right]\right\|^{2}
$$

where $\boldsymbol{P}_{c}$ is an $N \times N$ diagonal weighting matrix. The modification is tantamount to replacing $C_{s o}$ and $\boldsymbol{C}_{d o}$ by $\boldsymbol{P}_{c} \boldsymbol{C}_{s o}$ and $\boldsymbol{P}_{c} \boldsymbol{C}_{d o}$, respectively, in (21). With properly selected $\boldsymbol{P}_{c}$, we can control the size of the resulting phase perturbation for each of the center weights. A tradeoff that should be taken into account is that suppressing phase adaptation will result in a poorer approximation between the synthesized and desired patterns. Also, working with small phase perturbations increases the cost of the system due to the need of highprecision phase shifters.

Although the $2 J$ phase perturbations on the tail weights are allowed to vary freely, they are typically negligible compared to the amplitude perturbations under moderately good conditions, i.e., a small number of interferers from the sidelobe region. In this case, it is possible to work with amplitudeonly tail weights without severely distorting the synthesized patterns. Similar to the modification in (27), we can apply a $J \times J$ weighting matrix $\boldsymbol{P}_{r}$ on the subvector $\operatorname{Im}\{\boldsymbol{r}\}$. By choosing the diagonal components of $\boldsymbol{P}_{r}$ to be large enough, we can "turn off" the phase adaptation on $r$. In such a mode of operation, high-precision phase shifters are not needed for the tail weights. The reduction in system complexity with the mixed amplitude/phase-only nulling scheme is more significant as the size of the array increases. 


\section{Effects of Phase Errors}

In practice, the synthesized patterns are distorted by the errors in the adaptive weights. These errors can result from the deviation in element localizations, mutual coupling, and quantization effects in using digitally controlled attenuators and phase shifters. This section evaluates the effect of phase errors on the nulling performance of the adaptive array. Let $\boldsymbol{w}$ (either $\boldsymbol{s}$ or $\boldsymbol{d}$ ) be the weight vector subject to phase errors and $\overline{\boldsymbol{w}}$ the error-free weight vector. Denote as $\delta \phi$ the $M \times 1$ vector composed of the phase errors. Assuming that these phase errors are small and following (6), we have

$$
\boldsymbol{w} \approx \overline{\boldsymbol{w}}+j \overline{\boldsymbol{W}} \delta \boldsymbol{\phi}
$$

where $\overline{\boldsymbol{W}}=\operatorname{Diag}\{\overline{\boldsymbol{w}}\}$. The corresponding pattern at a prescribed interfering direction $u_{k}$ is given by

$$
w\left(u_{k}\right)=\boldsymbol{w}^{H} \boldsymbol{a}\left(u_{k}\right) \approx-j \delta \boldsymbol{\phi}^{T} \overline{\boldsymbol{W}}^{H} \boldsymbol{a}\left(u_{k}\right)
$$

where we invoked $\bar{w}\left(u_{k}\right)=\overline{\boldsymbol{w}}^{H} \boldsymbol{a}\left(u_{k}\right)=0$ since $u_{k}$ is a null of the error-free pattern. We observe from (29) that the effect of a phase error on $w\left(u_{k}\right)$ is enhanced by the corresponding amplitude weight. Also, $\left|w\left(u_{k}\right)\right|$ is small if the phase errors are approximately equal. This is, of course, not a realistic situation.

Another effect of phase errors is the change in the position of a null. Suppose that the original null at $u_{k}$ is shifted to $u_{k}+\delta u$ by a small amount $\delta u$. Using the first-order approximation, we get

$$
w\left(u_{k}+\delta u\right) \approx w\left(u_{k}\right)+w^{\prime}\left(u_{k}\right) \delta u \approx 0
$$

where $w^{\prime}(u)$ denotes the derivative of $w(u)$ with respect to $u$. Solving (30) for $\delta u$ gives

$$
\delta u \approx-\frac{w\left(u_{k}\right)}{w^{\prime}\left(u_{k}\right)}=\frac{j \delta \boldsymbol{\phi}^{T} \overline{\boldsymbol{W}}^{\boldsymbol{H}} \boldsymbol{a}\left(u_{k}\right)}{w^{\prime}\left(u_{k}\right)} .
$$

It is noteworthy that the solution in (31) may not be real. In this case, there is actually no null formed in the vicinity of $u_{k}$. That is, the original null has been "filled." A sufficient condition for $\delta u$ to be real is that $\delta \phi$ is antisymmetric such that $\boldsymbol{w}$ is conjugate symmetric. This in turn ensures that both $w(u)$ and $w^{\prime}(u)$ are real. Antisymmetric phase errors can be obtained if they arise from the quantization effect of using digital phase shifters.

The performance of an adaptive array using digital phase shifters has been discussed [9]. It is shown that nulling with full phase-only adaptation based on the small perturbation assumption is not reliable if low-precision phase shifters are used. This is because the small phase perturbations obtained with the algorithm cannot change the state of low-precision phase shifters. To remedy this, partial phase-only adaptation was suggested as a means of obtaining large phase perturbations. The previously described weighting method provides an alternative way to tackle the problem of working with lowprecision phase shifters. By turning off the phase adaptation on the tail weights and imposing a small weighting factor on the center weights, large phase perturbations can be obtained. However, care must be taken to avoid severe distortions of the synthesized patterns.

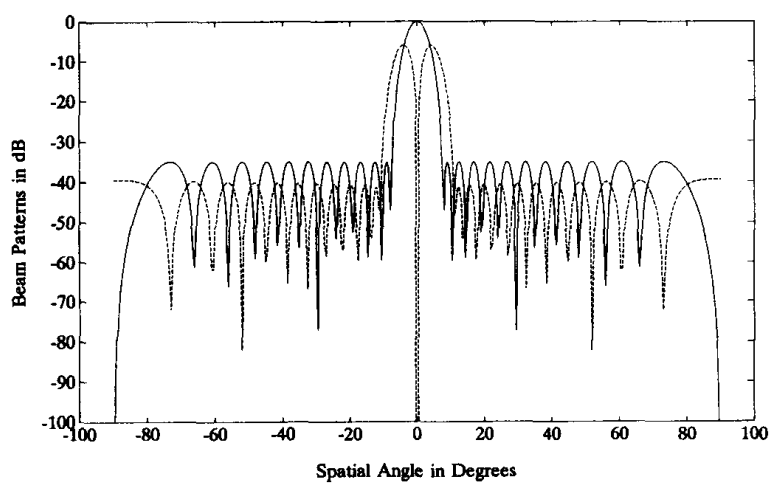

Fig. 2. Superposition of the normalized Chebyshev and Bayliss patterns with $-35 \mathrm{~dB}$ sidelobes synthesized with a 24-element ULA. Solid line: Chebyshev; dashed line: Bayliss.

\section{NUMERICAL EXAMPLES}

Computer simulations were conducted to ascertain the effectiveness of the proposed LS-fit based procedure for pattern synthesis. The ULA used was composed of 24 identical elements with a half-wavelength interelement spacing. The sidelobe levels of the desired Chebyshev and Bayliss patterns were the same equal to $-35 \mathrm{~dB}$. Fig. 2 shows the desired patterns in $\mathrm{dB}$ scale. Note that we have normalized the corresponding weight vectors, $\boldsymbol{s}_{o}$ and $\boldsymbol{d}_{o}$, so as to have a "hard" shared weights at the 6th and 19th elements, regardless of the actual value of $J$. For $J<6$, the extra shared phaseonly weights may be considered as offered by the degree of freedom in scaling the weight vectors.

The first set of examples demonstrates how the synthesized patterns change with the numbers of shared tail weights. The estimated interfering directions were $-45^{\circ}, 35^{\circ}$, and $50^{\circ}$. In this case, no weighting matrixes were applied in the objective function. The patterns synthesized with the procedure described in Section III are shown in Fig. 3 for $J=0,4$, 6 , and 8 . It is observed that the approximation between the synthesized and desired patterns improved as $J$ was increased from 0 to 6 , but degraded with $J=8$. This confirms our earlier statement that the appropriate number of shared weights is $2 J=\frac{M}{2}=12$. For $J=0$, there were actually no variable amplitude weights used and the patterns were synthesized solely with phase adaptation. Although it is simple to implement, working with full phase-only weights leads to poor nulling effect, as can be seen by comparing the depths of the nulls formed at the interfering directions in Figs. 3(a) and (c).

The second set of examples investigates the effect of using different weighting matrixes in the modified objective function of (27). The estimated interfering directions were the same as those given previously. The number of shared tail weights was fixed at $2 J=12$. In the first case, the tail weights were not weighted, and the center weights were weighted with $\boldsymbol{P}_{c}=$ $5 I_{6}$ and $1000 I_{6}$. Comparing the resulting patterns shown in Fig. 4(a) and (b) with Fig. 3(c) (corresponding to $P_{c}=I_{6}$ ), we find that the nulls formed at the interfering directions were deeper as the center weights were more emphasized. To 


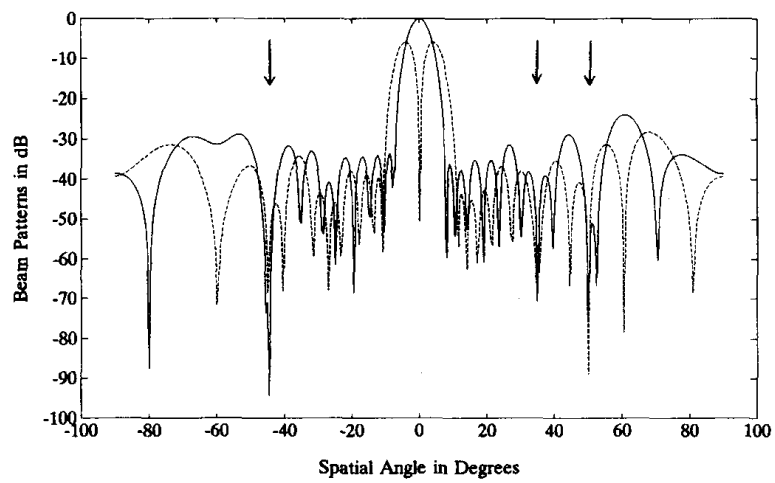

(a)

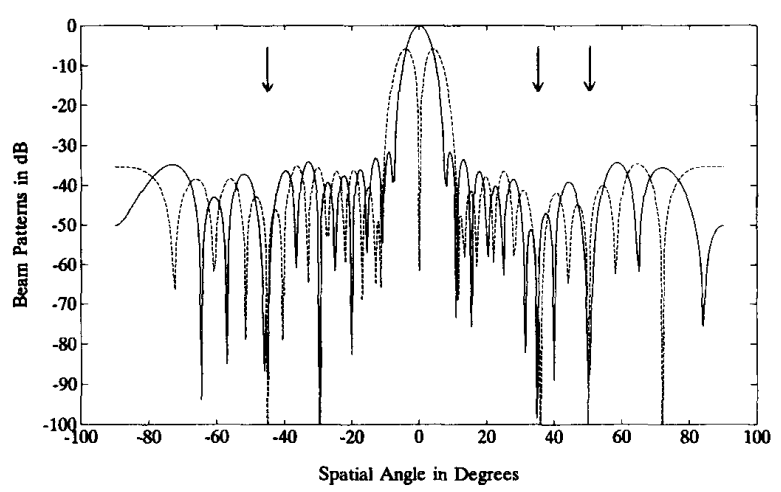

(c)

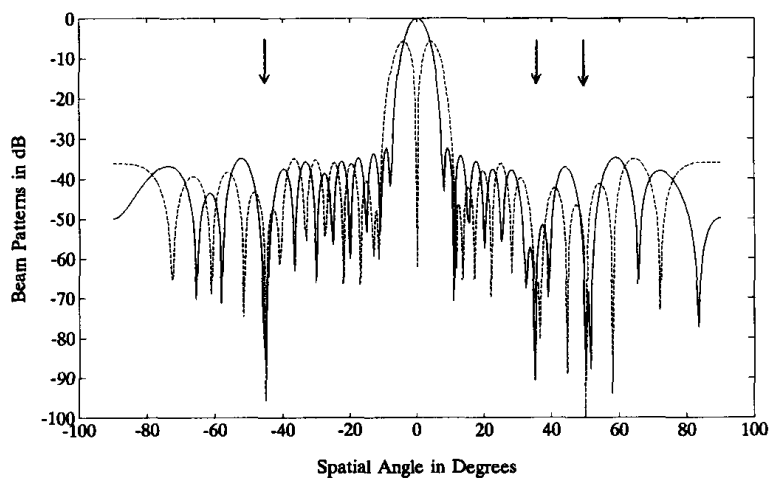

(b)

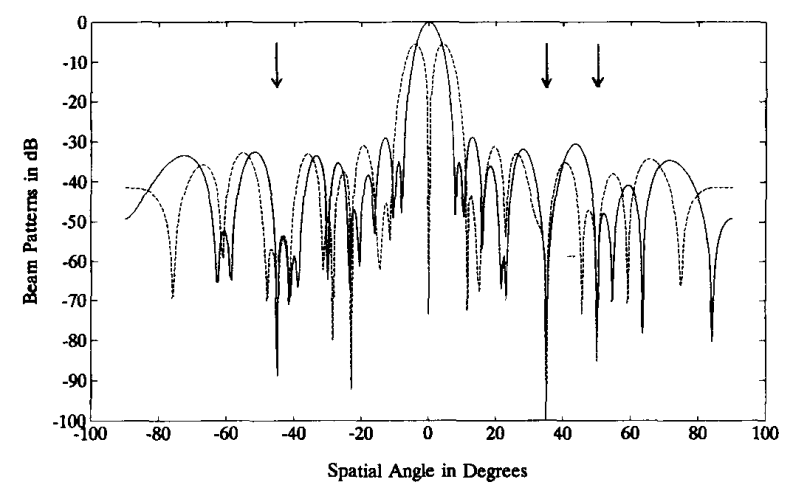

(d)

Fig. 3. Superposition of the LS-fit low-sidelobe patterns synthesized with a 24-element ULA for different number of shared tail weights. (a) $J=0$. (b) $J=4$. (c) $J=6$. (d) $J=8$. Solid line: sum; dashed line: difference. The interfering directions are $-45^{\circ}, 35^{\circ}$, and $50^{\circ}$.

see this, we note that a large weighting factor for the center weights in the LS-fit objective function leads to small phase perturbations, which in turn means that the error incurred with the approximation in (6) is reduced. As a result, the accuracy of nulling angles was improved. Note that with $\boldsymbol{P}_{c}=1000 \boldsymbol{I}_{6}$, the phase adaptation on the center weights were essentially turned off, leading to a system completely controlled by the shared tail weights. On the other hand, comparing the entire patterns shown in the three plots indicates that a small weighting factor for the center weights yields a better approximation between the synthesized and desired patterns. This is consistent with our earlier assertion that incorporating phase-only adaptation offers a larger degree of freedom for the LS-fit problem. In the second case, the center weights were not weighted, and the phase adaptation on the tail weights was turned off with $\boldsymbol{P}_{r}=1000 \boldsymbol{I}_{6}$ applied on $\operatorname{Im}\{\boldsymbol{r}\}$. We observe that low sidelobes were retained by the resulting patterns, shown in Fig. 4(c). This confirms the effectiveness of using amplitudeonly tail weights against sidelobe interference.

The final set of examples examines the nulling capability of the proposed method against extended interference. In the assumed scenario, an interfering source occupied the angular interval $\left[31^{\circ}, 40^{\circ}\right]$. Four point nulls were formed at $31^{\circ}, 34^{\circ}$, $37^{\circ}$, and $40^{\circ}$ to generate an effective broad null over the interfered region. The patterns obtained with the unweighted objective function are shown in Fig. 5(a), with $J=6$. We observe that the sidelobe level in the interfered region was about $-70 \mathrm{~dB}$ relative to the mainlobe peak of the sum pattern. The sidelobes outside the interfered region were kept pretty flat, though a little higher than the desired $-35 \mathrm{~dB}$. As a comparison, we also show in Fig. 5(b) and (c) the results obtained with $\left(\boldsymbol{P}_{c}, \boldsymbol{P}_{r}\right)=\left(1000 \boldsymbol{I}_{6}, \boldsymbol{I}_{6}\right)$ and $\left(\boldsymbol{I}_{6}, 1000 \boldsymbol{I}_{6}\right)$, respectively. We note that the patterns were severely distorted with the phase adaptation on the center weights turned off. On the other hand, working with amplitude-only tail weights did not degrade the results much. These indicate that incorporating phase adaptation on the center weights is more critical in dealing with complicated interference.

\section{CONCLUSION}

A simple LS-fit based method was proposed for the synthesis of low-sidelobe adaptive sum and difference patterns for linear arrays. The new method exploited the fact that the tail portions of the Chebyshev and Bayliss tapers are 


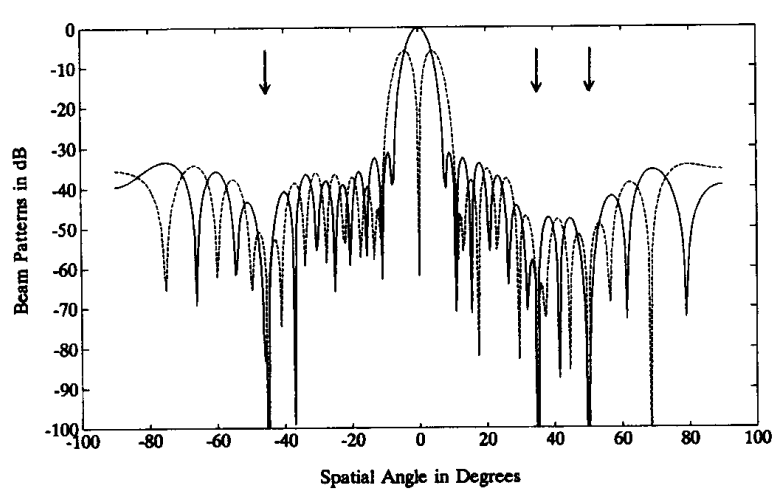

(a)

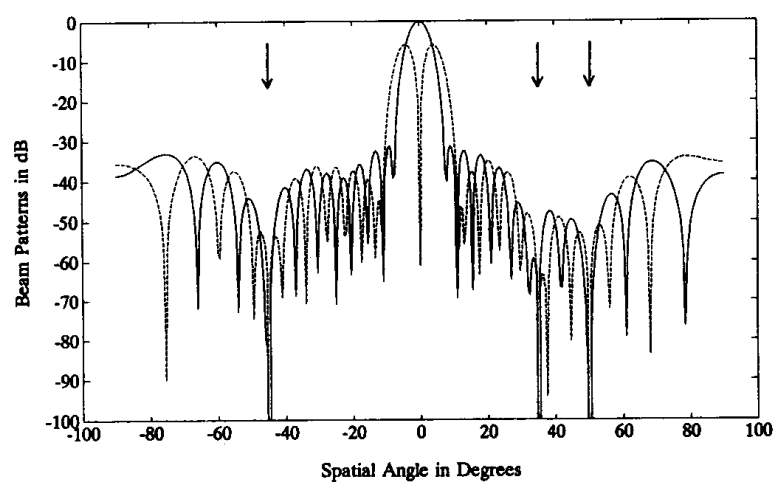

(b)

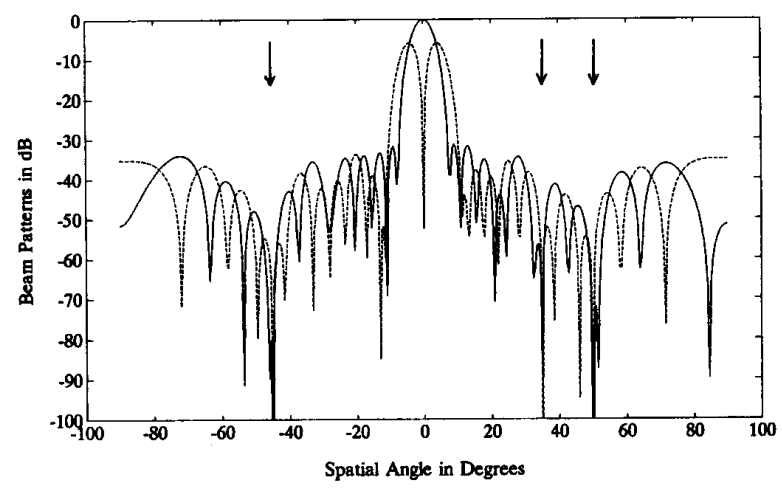

(c)

Fig. 4. Superposition of the LS-fit low-sidelobe patterns synthesized with a 24-element ULA using different weighting factors in the objective function. (a) $\boldsymbol{P}_{c}=5 I_{6}$. (b) $\boldsymbol{P}_{c}=1000 I_{6}$. (c) $\boldsymbol{P}_{r}=1000 I_{6}$. Solid line: sum; dashed line: difference. The interfering directions are $-45^{\circ}, 35^{\circ}$, and $50^{\circ} . J=6$ for all cases.

similar in shape such that the sum and difference beamformers producing the desired low-sidelobe patterns can in fact share a set of common tail weights. It was found that the appropriate choice is that one-fourth of the weights from both ends of the aperture are shared. System complexity was further eased by forcing the amplitudes of the center portions of the sum

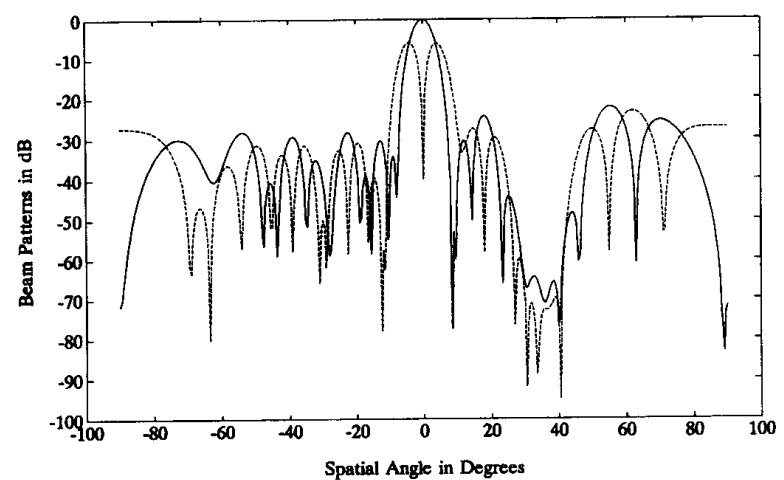

(a)

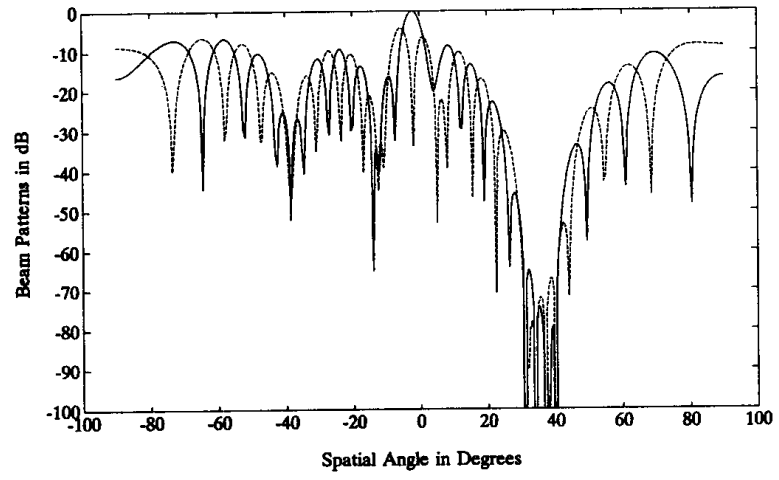

(b)

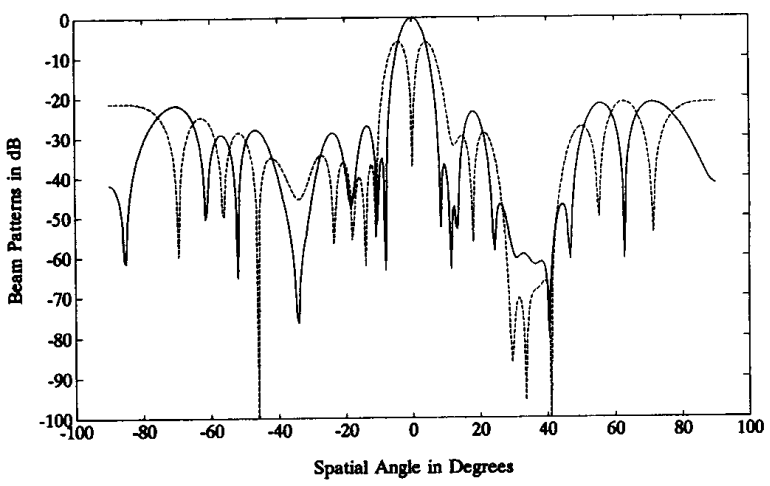

(c)

Fig. 5. Superposition of the LS-fit low-sidelobe patterns synthesized with a 24-element ULA using different weighting factors in the objective function. (a) Unweighted. (b) $\boldsymbol{P}_{c}=1000 I_{6}$. (c) $\boldsymbol{P}_{r}=1000 I_{6}$. Solid line: sum; dashed line: difference. Four nulls are formed at $31^{\circ}, 34^{\circ}, 37^{\circ}$, and $40^{\circ}$ to produce a broad null. $J=6$ for all cases.

and difference weights to be fixed. As a result, the number of variable amplitude weights needed was reduced from $2 M$ to $\frac{M}{2}$, where $M$ is the number of array elements. Phase-only adaptation was incorporated on the unshared center weights to recover the degree of freedom in pattern synthesis. By using different weighting factors in the LS-fit objective function, 
several configurations of the adaptive weights were obtained that exhibited different tradeoffs between performance and complexity. Numerical examples demonstrated that the proposed method was effective in combating both point and extended interference.

\section{REFERENCES}

[1] R. L. Haupt, "Simultaneous nulling in the sum and difference patterns of a monopulse antenna," IEEE Trans. Antennas Propagat., vol. 32, pp. 486-493, May 1984.

[2] H. Steyskal, R. A. Shore, and R. L. Haupt, "Methods for null control and their effects on the radiation pattern," IEEE Trans. Antennas Propagat., vol. 34, pp. 404-409, Mar. 1986.

[3] C. Baird and G. Rasswiler, "Adaptive sidelobe nulling using digitally controlled phase shifters," IEEE Trans. Antennas Propagat., vol. 24 pp. 638-649, Sept. 1976.

[4] H. Steyskal, "Simple method for pattern nulling by phase perturbation," IEEE Trans. Antennas Propagat., vol. 31, pp. 163-166, Jan. 1983.

[5] T. B. Vu, "Simultaneous nulling in sum and difference pattern by amplitude control," IEEE Trans. Antennas Propagat., vol. 34, pp. 214-218, Feb. 1986.

[6] R. L. Haupt, "Null synthesis with phase and amplitude controls at the subarray outputs," IEEE Trans. Antennas Propagat., vol. 33, pp. 505-509, May 1985.
[7] C. L. Dolph, "A current distribution for broadside arrays which optimizes the relationship between beamwidth and side lobe level,' Proc. IRE, vol. 34, pp. 335-348, 1946.

[8] E. T. Bayliss, "Design of monopulse antenna difference patterns with low side lobes," Bell System Tech. J., vol. 47, pp. 632-640, 1968.

[9] D. C. Chang, K. T. Ho, and C. I. Hung, "Partial adaptive nulling on a monopulse phased array antenna system," IEEE Trans. Antennas Propagat., vol. 40, pp. 121-125, Feb. 1992.

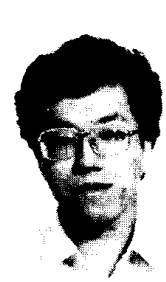

Ta-Sung Lee (M'94) was born in Taipei, Taiwan, Republic of China, on october 20,1960. He received the B.S. degree from National Taiwan University in 1983, the M.S. degree from the University of Wisconsin, Madison, in 1987, and the Ph.D. degree from Purdue University, West Lafayette, IN, in 1989 , all in electrical engineering.

From 1987 to 1989 , he was a David Ross Graduate Research Fellow at Purdue University. In 1990 he joined the faculty of National Chiao Tung University, Taiwan, where he currently holds a position as Associate Professor in the Department of Communicaiton Engineering. His present research interests include sensor array signal processing, adaptive antennas for mobile communications, underwater acoustic signal processing, and medical imaging.

Dr. Lee is a member of Phi Tau Phi 